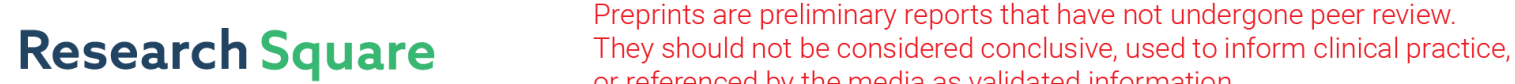 or referenced by the media as validated information. \\ Identification of IncRNA-miRNA-mRNA regulatory network associated with Primary Open Angle Glaucoma
}

\section{Minwen Zhou}

Shanghai Jiaotong University First People's Hospital

\section{Bing Lu}

shanghai general hospital

Wei Tan

zunyi first people's hospital

Mingshui Fu ( $\nabla$ fumingshui@126.com )

Shanghai General Hospital

Research article

Keywords: Primary open angle glaucoma, long noncoding RNA, IncRNA-miRNA-mRNA regulatory network

Posted Date: February 13th, 2020

DOI: https://doi.org/10.21203/rs.2.19179/v2

License: (c) (i) This work is licensed under a Creative Commons Attribution 4.0 International License. Read Full License 


\section{Abstract}

Background: Primary open angle glaucoma (POAG) is a multifactorial disorder characterized by a progressive permanent degeneration of retinal ganglion cell (RGCs) death. An increasing number of studies have suggested that long noncoding RNAs (IncRNAs) have the ability to regulate gene expression; however, thus far, the mechanisms and functions of IncRNAs in the development of POAG are still unclear. Methods: Using the data from Gene Expression Omnibus (GEO), differentially expressed IncRNAs and differentially expressed mRNAs between POAG patients and controls were identified. Then, the IncRNA-miRNA-mRNA competing endogenous RNA (ceRNA) network was constructed, and the key IncRNAs in POAG were identified. A Gene Ontology (GO) analysis and a Kyoto Encyclopedia of Genes and Genomes (KEGG) pathway analysis were performed to assess the enriched biological functions of mRNA in the ceRNA network.

Results: During this study, a POAG-related ceRNA network with 37 miRNA nodes, 248 IncRNA nodes, 178 mRNA nodes, and 1985 edges was constructed. In addition, four IncRNAs (DNAJC27-AS1, AF121898, OIP5-AS1, and SNX29P2) were established as hub RNAs in this ceRNA network. The functional assay showed that $18 \mathrm{GO}$ terms and 17 pathways were enriched.

Conclusion: This study provides novel insights into the IncRNA-related ceRNA network in POAG, and the four IncRNAs were identified in the development of POAG.

\section{Background}

Primary open angle glaucoma (POAG), a frequent type of glaucoma worldwide, is a complex characterized by the progressive permanent degeneration of retinal ganglion cell (RGCs) death and distinctive visual field loss[1, 2] . Regarding disease etiology, multiple risk factors, such as age[3, 4], elevated intraocular pressure (IOP) [5, 6], family history[7], and ethnic background[8], have been established in connection with the risk of POAG. In addition to these risk factors, genetics has also been shown to play a key role in the pathogenesis of POAG[9-11]. Nevertheless, the actual molecular mechanisms are still poorly understood.

A non-coding RNA (ncRNA) is an RNA molecule that is not translated into a protein. The ncRNA molecule has been found to be involved in master regulators in various biological pathologic processes[12, 13]. Long noncoding RNA (IncRNA) is a typical ncRNA with a non-protein-coding function exceeding 200 nucleotides. LncRNA has the ability to regulate gene expression by participating in regulating the transcription and translation of genes[14, 15]. Recently, emerging evidence has suggested the critical role of IncRNAs in the occurrence of POAG[9, 10, 16]; however, thus far, the mechanisms and functions of most IncRNAs remain incomprehensible with only a small portion being well-annotated.

Recently, it has been suggested that IncRNAs are emerging as competing endogenous RNAs (ceRNAs) to communicate with messenger RNA (mRNA) through competitive microRNA (miRNA). Ordinarily, miRNAs can induce target gene degradation or can inhibit mRNA translation[17]; however, IncRNA can share 
miRNA response elements (MREs) with mRNA and can then alleviate the inhibition of the miRNAmediated target gene[17]. Thus, it is a competent method used to understand the complex functions of IncRNA by understanding its relationship with microRNAs and mRNAs due to the annotated functions of miRNAs and mRNAs. Thus, bioinformatic methods were carried out to analyze the gene expression profile in data sets obtained from the Gene Expression Omnibus (GEO) for the aim to further determine an innovative regulatory mechanism based on the IncRNA-miRNA-mRNA ceRNA theory in the development of POAG.

\section{Methods}

\section{Sample}

Samples for experimental groups were obtained from POAG patients who did not receive any glaucoma medication 4 months before surgery and had uncontrolled IOP. The control group for AH profiling consisted of age- and sex-matched age-related cataract patients who were candidates for cataract surgery. The mean age in the control group is $63.2 \pm 3.5$ years and in the POAG group it is $61.0 \pm 2.9$ years. The IOP were $18.8 \pm 0.7$ and $27.8 \pm 2.1 \mathrm{mmHg}$ in the control and POAG groups, respectively. The proportion of males was $48.8 \%$ and $44.2 \%$ in the control and POAG groups, respectively. Aqueous humor (approximately $100 \mathrm{~mL}$ ) was carefully collected from patients who underwent surgery by paracentesis of the anterior chamber, using a 27-gauge needle inserted through the peripheral cornea under a microscope. During paracentesis, the needle was kept away from touching the iris and lens. All samples were immediately cooled at $-80^{\circ} \mathrm{C}$ and protected from light in a dry place until they were measured.

\section{Expression profiles of IncRNAs and mRNAs}

Using the Agilent-079487 Arraystar Human LncRNA microarray platform (GPL21827), mRNA and IncRNA expression data of GSE101727 were obtained from the publicly available GEO database (http://www.ncbi.nlm.nih.gov/geo/). The database consisted of the disease-related extracellular IncRNAs and mRNAs in the aqueous humor (AH) of individual POAG patients and the AH of individual cataracts as the control.

\section{Probe re-annotation}

The raw data of GSE101727 only supports the sequence data format and not the gene symbol. Thus, using the software of perl (https://www.perl.org/; version 5.30.0), the sequence data format was converted to the FASTA format first. The human genome and related annotation file were obtained from the GENCODE database (https://www.gencodegenes.org). Then, the sequence alignment methods were used via NCBI-blast software (version 2.7.1). Subsequently, the probeMatrix file was converted to the gene symbol matrix file, and genes were classified as protein-coded RNA and IncRNA using the perl software.

\section{Differential analysis of RNAs}


To establish differentially expressed IncRNA and mRNA, the limma package of the R Software for statistical analysis was used. This $R$ package was also used to calculate the $\log _{2}$ fold change $\left(\log _{2} F C\right)>$ 1 and the false discovery rate (FDR). An adjusted $P<0.05$ was used as the standard. The limma and pheatmap packages were used to draw volcano plots and heatmaps, respectively.

\section{Identification of potential ceRNA interactions}

Both mRNAs and IncRNAs that were negatively correlated with certain common miRNAs were defined as candidate ceRNA pairs. The mircode online tool (http://www.mircode.org/) was used to predict the IncRNA and miRNA interaction. The putative miRNA-mRNA interactions were collected from miRDB (http://mirdb.org/), TargetScan (http://www.targetscan.org/vert_72/), and miRTarBase (http://mirtarbase.mbc.nctu.edu.tw/php/index.php) databases. Only miRNA-mRNA interactions predicted by all three databases were included. Thus, all the possible miRNA targets were predicted using the perl software tool.

\section{Construction of the IncRNA-mediated ceRNA network}

The IncRNA-mediated ceRNA network was constructed on the basis that ceRNA can bind to miRNA through MREs. This ceRNA network was visualized using Cytoscape software (Version 3.6.0).[18] In this network, nodes and edges represented biological data in a direct manner in which each node represented a biological molecule, and the edges represented interactions between nodes.[19] LncRNAs, mRNAs, and miRNAs in the ceRNA network were presented as blue diamonds, green ellipses, and red triangles, respectively. In addition, the topological features of this ceRNA network was calculated by a built-in NetworkAnalyzer tool in Cytoscape software, including betweenness, network degree, and closeness centrality.[20] These topological parameters are standard measures of centrality in a network.

Betweenness centrality was calculated as the number of shortest paths between all pairs of nodes in the network that passed through the node. The degree centrality was calculated as the number of edges linked to a node. The closeness centrality of a node was the shortest path between a node and other nodes.[20] To compare the differences in the degree, closeness, and betweenness centrality among IncRNAs, miRNAs, and mRNAs, the Kruskal-Wallis test was used.

\section{Gene Ontology and KEGG pathway analysis}

For the Gene Ontology (GO) and the Kyoto Encyclopedia of Genes and Genomes (KEGG) pathwayenrichment analysis, the clusterProfilerGO and clusterProfilerKEGG packages of the $\mathrm{R}$ Software were used. $P<0.05$ was used as the cutoff criterion.

\section{Results}

\section{Data set acquisition and identification of differentially expressed RNAs}


From the GEO database, $10 \mathrm{AH}$ samples from POAG patients and $10 \mathrm{AH}$ samples from cataract patients were collected. After selecting the pre-treated data based on the adjusted $P<0.05$ and $\log _{2}$ FC $>1$ change, between the 10 patients' samples and the 10 control samples, a total of 4,130 differentially expressed RNAs were identified. Among them, 1,041 were IncRNA (508 up- and 533 down-regulated), and 3,089 were mRNA (2135 up- and 954 down-regulated). Volcano plots of all differentially expressed genes were generated (fig. 1). The heat maps of IncRNAs, mRNAs, and all RNAs showed the differences between POAG patients and the control group (fig. 1).

\section{ceRNA network construction and analysis}

The differentially expressed IncRNAs established were selected using the miRcode online tool, and the potential predicted target miRNAs were compared. Then, the relationships between differentially expressed mRNAs and miRNAs were also evaluated by the miRTarBase, MiRDB, and Targetscan databases. Finally, 248 POAG-specific IncRNAs that putatively targeted 37 POAG-specific miRNAs and the comparisons of 37 POAG-specific miRNAs and 178 POAG -specific mRNAs were involved in the ceRNA network. As displayed in fig. 2, 37 miRNA nodes, 248 IncRNA nodes, 178 mRNA nodes, and 1,985 edges comprised the IncRNA-miRNA-mRNA network. The topological features of this ceRNA network were assessed by a built-in NetworkAnalyzer tool in Cytoscape software, including betweenness, network degree, and closeness centrality. Generally, the nodes in the ceRNA network with a higher degree, closeness, and betweenness centrality demonstrated a higher possibility of hub nodes in the ceRNA network. A Venn diagram for the overlapping top 40 genes with topological features in each dimension was created (Table 1, fig. 3D). Finally, nine miRNAs (hsa-miR-20b-5p, hsa-miR-761, hsa-miR-17-5p, hsamiR-338-3p, hsa-miR-24-3p, hsa-miR-125b-5p, hsa-miR-3619-5p, hsa-miR-129-5p, and hsa-miR-27a) and four IncRNAs (DNAJC27-AS1, AF121898, OIP5-AS1, and SNX29P2) were established as hub RNAs in the ceRNA network. When comparing the differences in the degree, closeness, and betweenness centrality among IncRNAs, miRNAs, and mRNAs, the result showed that IncRNAs and miRNAs had a higher degree, closeness, and betweenness centrality than mRNAs (fig. 3), indicating that IncRNAs and miRNAs tended to be pivotal to the risk of POAG.

\section{Functional annotation of the ceRNA network}

To achieve a more thorough understanding of the mRNAs' function in the ceRNA network, a GO and a KEGG analysis were performed using R software. The functional assay showed that $18 \mathrm{GO}$ terms and 17 pathways were enriched. For the GO analysis, POAG was significantly enriched in ubiquitin-like protein ligase binding, ubiquitin protein ligase binding, transcription factor activity, etc. The KEGG-enriched analysis results indicated that major pathways, including the mitogen-activated protein kinase (MAPK) signaling pathway, endocytosis pathway, and Wnt signaling pathway, were involved in these mRNAs. The results of the GO and KEGG enriched analyses are displayed in fig. 4.

\section{Discussion}


POAG is a multifactorial disorder with various etiologies that is estimated to occur in about 52 million patients in 2020 around the world[21]. During the last few years, considerable efforts have been made to investigate the molecular mechanisms of POAG[22, 23]; however, most previous studies have mainly focused on protein-coding genes or miRNAs but not on IncRNAs[22-24].

It has been reported that more than 10,000 IncRNAs are produced by human genomes; however, to date, little information related to IncRNAs, especially their function, have been established[25, 26]. Recently, IncRNA has been found to be involved in some important regulatory processes, including transcriptional interference, transcriptional activation, and chromatin modification, and it may serve as a biomarker for various diseases, such as lung cancer[27], colorectal cancer[28], diabetes mellitus[29], and liver fibrosis[30]. Nevertheless, few studies have reported the role of IncRNA in the pathogenesis of POAG. Thus, the identification of IncRNA in the pathogenesis of POAG is necessary.

The newly emerged ceRNA hypothesis has been suggested as an innovative post-transcriptional regulatory mechanism of gene expression. Under this ceRNA network, the IncRNAs and mRNAs are connected by their common target miRNAs[30]. In recent years, several studies have explored the underlying molecular mechanisms based on the ceRNA network in some diseases, such as breast cancer[32], ischemic stroke[33], and rheumatoid arthritis[34]. To identify IncRNAs significantly associated with POAG, in this study, the mRNA and IncRNA expression profiles of POAG patients were first used and combined with miRNA-target interactions to create a ceRNA network and to investigate the potential implications of these IncRNAs in the development of POAG.

In this study, four IncRNAs (DNAJC27-AS1, AF121898, OIP5-AS1, and SNX29P2) served as the hub nodes finally. In addition, the GO and KEGG pathway analyses were used to assess enriched biological functions. The differentially expressed mRNA in the IncRNA-miRNA-mRNA ceRNA network-related GO analysis showed that ubiquitin-like protein ligase, ubiquitin protein ligase, and others could play an important role in the development of POAG. The pathway analysis further revealed that 17 unique pathways were enriched, including the MAPK signaling pathway, endocytosis pathway, and Wnt signaling pathway. In fact, an increasing amount of experimental evidence has indicated that these enriched pathways have always been involved in glaucoma. For example, Beit-Yannai et al[35]. found that $\mathrm{AH}$ in a rat model of induced elevated IOP expressed several signaling members of the MAPK family; they suggested that MAPKs present in the aqueous humor are a novel signal involved in glaucoma pathology. In addition, another study showed that the MAPK signal pathway participates in protecting human trabecular meshwork cells from pressure-induced apoptosis[36]. Webber et al.[37] reported that Wnt signaling pathways play important roles in the regulation of TM homeostasis and IOP[38]. Thus, the enrichment results could suggest that the IncRNA-miRNA-mRNA ceRNA network plays an important role, by way of these pathways, in the development of POAG.

Hub nodes, which have been examined in some studies and are characterized by their high degree of connectivity to other nodes in the ceRNA network, can be used as topological properties of the ceRNA network to assess the significance of genes[39, 40]. In the present study, four IncRNAs (DNAJC27-AS1, 
AF121898, OIP5-AS1, and SNX29P2) were observed to be topological hub nodes whose betweenness, network degree, and closeness centrality were significantly higher in comparison with other IncRNAs. Thus far, among these hub IncRNAs, DNAJC27-AS1, AF121898, and SNX29P2 have not been reported in any study. OIP5-AS1 is an antisense IncRNA that has been reported to play a critical role in various disorders, including oral squamous cell carcinoma[41], gastric cancer[42], cardiovascular disease[43], and multiple sclerosis[44]. Li and colleague's study revealed that OIP5-AS1 could promote the progression of oral squamous cell carcinoma by regulating the miR-338-3p/NRP1 axis[41]. Another study has also indicated an aggressive role of ceRNA to drive migration, invasion, and proliferation of human hemangioma endothelial cells via regulating the miR-195-5p/NOB1 axis[45]. In the present study, it was observed that OIP5-AS1 displayed low-expression, which could compete with miRNAs (hsa-miR-17-5p, hsa-miR-20b-5p, hsa-miR-761, hsa-miR-3619-5p, hsa-miR-24-3p, hsa-miR-27a, hsa-miR-338-3p, and hsamiR-129-5p) to regulate target gene expression. These miRNAs interacted with OIP5-AS1 and have been known to be involved in glaucoma. For example, Zhao's study has confirmed that miR-27a (a target miRNA of OIP5-AS1) has protective impacts on H2O2-injured human trabecular meshwork cells, which comprise a common glaucoma cell model[46]. Another miRNA (miR-17-5p, a target miRNA of OIP5-AS1) has been found to have the function of regulating the proliferation and apoptosis of human trabecular meshwork cells in response to oxidative stress[47]. Up to now, the research on OIP5-AS1 in glaucoma is still blank. The findings of our study show that OIP5-AS1 may be related to the development of glaucoma via IncRNA-miRNA-mRNA ceRNA network analysis. Therefore, the OIP5-AS1/miRNA/mRNA axis may become a hot issue for the study of glaucoma in the future.

Although the findings of this study have important clinical significance, the limitations should be discussed. First, the conclusion of this study based on the GEO database should be verified by other experimental evidence. Second, the precise cellular sources and mechanisms underlying hub genes, such as OIP5-AS1, DNAJC27-AS1, AF121898, and SNX29P2, in relation to POAG should be further investigated. Third, from the GEO database, 10 AH samples from POAG patients and $10 \mathrm{AH}$ samples from cataract patients is a relatively small sample; this is the major drawback when performing genetic studies of association. Thus, the conclusion and the results of this study should be interpreted with caution.

In summary, during this study, a POAG-related IncRNA-miRNA-mRNA ceRNA network was constructed, and hub IncRNAs, such as OIP5-AS1, DNAJC27-AS1, AF121898, and SNX29P2, were identified in the development of POAG, which provided novel insights into exploring the underlying mechanism of POAG. Further experimental studies should be performed to elucidate the molecular mechanisms underlying the IncRNA function in POAG.

\section{Declarations}

Acknowledgments

Not applicable

\section{Availability of data and material}


The datasets used and/or analyzed during the current study are available from the corresponding author on reasonable request.

\section{Author Contributions}

M.F., W.T. and B. L designed the study, analyzed data; M.Z analyzed data and wrote the manuscript. All authors have read and approved the manuscript in its current state.

\section{Funding}

This research was supported by the Shanghai Natural Science Foundation (19ZR1440900), Shanghai Pujiang Talents Plan (2019PJD047), National Natural Science Foundation of China (81500714, 81660162), and Shanghai-Zunyi Diabetic ophthalmopathy Clinical Medical Science and Technology Innovation Center Projects [No. (2018)4]. The funding organizations had no role in designing or conducting of this Research.

Ethics approval and consent to participate Not applicable.Consent for publication Not applicable.Competing interests

The authors declare that they have no conflict of interest.

\section{Author details}

${ }^{1}$ Department of Ophthalmology, Shanghai General Hospital (Shanghai First People's Hospital), Shanghai Jiao Tong University School of Medicine, Shanghai, China

${ }^{2}$ Shanghai Key Laboratory of Fundus Diseases, Shanghai, China

${ }^{3}$ The Department of Ophthalmology, The Third Affiliated Hospital of Zunyi Medical University, 98 Feng huang Road, Zunyi

\section{Abbreviations}

POAG: Primary open angle glaucoma; RGC: retinal ganglion cell; IOP: intraocular pressure; ncRNA: noncoding RNA; IncRNA: long noncoding RNA; ceRNA: competing endogenous RNA; mRNA: messenger RNA; miRNA: microRNA; MRE: miRNA response element; GEO: Gene Expression Omnibus; AH: aqueous humor; $\log _{2} \mathrm{FC}$; $\log _{2}$ fold change; FDR; false discovery rate; GO: Gene Ontology; KEGG: Kyoto Encyclopedia of Genes and Genomes

\section{References}

1. Fingert JH: Primary open-angle glaucoma genes. Eye (Lond) 2011, 25(5):587-595.

2. Liu Y, Allingham RR: Molecular genetics in glaucoma. EXP EYE RES 2011, 93(4):331-339. 
3. Kim KE, Kim MJ, Park KH, Jeoung JW, Kim SH, Kim CY, Kang SW: Prevalence, Awareness, and Risk Factors of Primary Open-Angle Glaucoma: Korea National Health and Nutrition Examination Survey 2008-2011. OPHTHALMOLOGY 2016, 123(3):532-541.

4. Kreft D, Doblhammer G, Guthoff RF, Frech S: Prevalence, incidence, and risk factors of primary openangle glaucoma - a cohort study based on longitudinal data from a German public health insurance. BMC PUBLIC HEALTH 2019, 19(1):851.

5. Baskaran M, Foo RC, Cheng CY, Narayanaswamy AK, Zheng YF, Wu R, Saw SM, Foster PJ, Wong TY, Aung T: The Prevalence and Types of Glaucoma in an Urban Chinese Population: The Singapore Chinese Eye Study. JAMA OPHTHALMOL 2015, 133(8):874-880.

6. Kinouchi R, Ishiko S, Hanada K, Hayashi H, Mikami D, Tani T, Zenimaru T, Kawai M, Nakabayashi S, Kinouchi $\mathrm{M}$ et al: A low meat diet increases the risk of open-angle glaucoma in women-The results of population-based, cross-sectional study in Japan. PLOS ONE 2018, 13(10):e204955.

7. Jonas JB, Aung T, Bourne RR, Bron AM, Ritch R, Panda-Jonas S: Glaucoma. LANCET 2017, 390(10108):2183-2193.

8. Tham YC, Li X, Wong TY, Quigley HA, Aung T, Cheng CY: Global prevalence of glaucoma and projections of glaucoma burden through 2040: a systematic review and meta-analysis. OPHTHALMOLOGY 2014, 121(11):2081-2090.

9. Xie L, Mao M, Wang C, Zhang L, Pan Z, Shi J, Duan X, Jia S, Jiang B: Potential Biomarkers for Primary Open-Angle Glaucoma Identified by Long Noncoding RNA Profiling in the Aqueous Humor. AM J PATHOL 2019, 189(4):739-752.

10. Nunes HF, Ananina G, Costa VP, Zanchin N, de Vasconcellos J, de Melo MB: Investigation of CAV1/CAV2 rs4236601 and CDKN2B-AS1 rs2157719 in primary open-angle glaucoma patients from Brazil. OPHTHALMIC GENET 2018, 39(2):194-199.

11. Lu SY, He ZZ, Xu JX, Yang C, Chen LJ, Gong B: Association of Polymorphisms at the SIX1-SIX6 Locus With Primary Open-Angle Glaucoma. Invest Ophthalmol Vis Sci 2019, 60(8):2914-2924.

12. Huttenhofer A, Schattner P, Polacek N: Non-coding RNAs: hope or hype? TRENDS GENET 2005, 21(5):289-297.

13. Palazzo AF, Lee ES: Non-coding RNA: what is functional and what is junk? FRONT GENET 2015, 6:2.

14. Ponting CP, Oliver PL, Reik W: Evolution and functions of long noncoding RNAs. CELL 2009, 136(4):629-641.

15. Jiang W, Meng $\mathrm{K}$, Yang T: Long non-coding RNA PROX1-AS1 promotes the proliferation and migration in gastric cancer by epigenetically activating FGFR1. PANMINERVA MED 2019.

16. Pasquale LR, Loomis SJ, Kang JH, Yaspan BL, Abdrabou W, Budenz DL, Chen TC, Delbono E, Friedman DS, Gaasterland D et al: CDKN2B-AS1 genotype-glaucoma feature correlations in primary open-angle glaucoma patients from the United States. AM J OPHTHALMOL 2013, 155(2):342-353.

17. Salmena L, Poliseno L, Tay Y, Kats L, Pandolfi PP: A ceRNA hypothesis: the Rosetta Stone of a hidden RNA language? CELL 2011, 146(3):353-358. 
18. Yue B, Li H, Liu M, Wu J, Li M, Lei C, Huang B, Chen H: Characterization of IncRNA-miRNA-mRNA Network to Reveal Potential Functional ceRNAs in Bovine Skeletal Muscle. FRONT GENET 2019, 10:91.

19. Kohl M, Wiese S, Warscheid B: Cytoscape: software for visualization and analysis of biological networks. Methods Mol Biol 2011, 696:291-303.

20. Assenov Y, Ramirez F, Schelhorn SE, Lengauer T, Albrecht M: Computing topological parameters of biological networks. BIOINFORMATICS 2008, 24(2):282-284.

21. Tham YC, Li X, Wong TY, Quigley HA, Aung T, Cheng CY: Global prevalence of glaucoma and projections of glaucoma burden through 2040: a systematic review and meta-analysis.

OPHTHALMOLOGY 2014, 121(11):2081-2090.

22. Gong B, Zhang H, Huang L, Chen Y, Shi Y, Tam PO, Zhu X, Huang Y, Lei B, Sundaresan $P$ et al: Mutant RAMP2 causes primary open-angle glaucoma via the CRLR-CAMP axis. GENET MED 2019.

23. Shen T, Gupta VK, Klistorner A, Chitranshi N, Graham SL, You Y: Sex-Specific Effect of BDNF Val66Met Genotypes on the Progression of Open-Angle Glaucoma. Invest Ophthalmol Vis Sci 2019, 60(4):1069-1075.

24. Liu Y, Wang Y, Chen Y, Fang X, Wen T, Xiao M, Chen S, Zhang X: Discovery and Validation of Circulating Hsa-miR-210-3p as a Potential Biomarker for Primary Open-Angle Glaucoma. Invest Ophthalmol Vis Sci 2019, 60(8):2925-2934.

25. Fotuhi SN, Khalaj-Kondori M, Hoseinpour FM, Talebi M: Long Non-coding RNA BACE1-AS May Serve as an Alzheimer's Disease Blood-Based Biomarker. J MOL NEUROSCI 2019.

26. Jiang YF, Zhang HY, Ke J, Shen H, Ou HB, Liu Y: Overexpression of LncRNA GHET1 predicts an unfavourable survival and clinical parameters of patients in various cancers. J CELL MOL MED 2019, 23(8):4891-4899.

27. Liu HY, Lu SR, Guo ZH, Zhang ZS, Ye X, Du Q, Li H, Wu Q, Yu B, Zhai Q et al: IncRNA SLC16A1-AS1 as a novel prognostic biomarker in non-small cell lung cancer. J Investig Med 2019.

28. Zhao Y, Du T, Du L, Li P, Li J, Duan W, Wang Y, Wang C: Long noncoding RNA LINC02418 regulates MELK expression by acting as a ceRNA and may serve as a diagnostic marker for colorectal cancer. CELL DEATH DIS 2019, 10(8):568.

29. Zhang W, Zheng J, Hu X, Chen L: Dysregulated expression of long noncoding RNAs serves as diagnostic biomarkers of type 2 diabetes mellitus. ENDOCRINE 2019.

30. Zhang K, Han Y, Hu Z, Zhang Z, Shao S, Yao Q, Zheng L, Wang J, Han X, Zhang Y et al: SCARNA10, a nuclear-retained long non-coding RNA, promotes liver fibrosis and serves as a potential biomarker. THERANOSTICS 2019, 9(12):3622-3638.

31. Tay Y, Rinn J, Pandolfi PP: The multilayered complexity of ceRNA crosstalk and competition. NATURE 2014, 505(7483):344-352.

32. Fan CN, Ma L, Liu N: Systematic analysis of IncRNA-miRNA-mRNA competing endogenous RNA network identifies four-IncRNA signature as a prognostic biomarker for breast cancer. J TRANSL MED 2018, 16(1):264. 
33. Guo X, Yang J, Liang B, Shen T, Yan Y, Huang S, Zhou J, Huang J, Gu L, Su L: Identification of Novel LncRNA Biomarkers and Construction of LncRNA-Related Networks in Han Chinese Patients with Ischemic Stroke. CELL PHYSIOL BIOCHEM 2018, 50(6):2157-2175.

34. Jiang $H$, Ma R, Zou S, Wang $Y$, Li Z, Li W: Reconstruction and analysis of the IncRNA-miRNA-mRNA network based on competitive endogenous RNA reveal functional IncRNAs in rheumatoid arthritis. MOL BIOSYST 2017, 13(6):1182-1192.

35. Beit-Yannai $E$, Shmulevich A: Does the aqueous humor have a role in mitogen-activated protein kinase (MAPK) intracellular signaling in Glaucoma? MED HYPOTHESES 2007, 68(2):299-302.

36. Meng B, Li H, Sun X, Qu W, Yang B, Cheng F, Shi L, Yuan H: sigma-1 receptor stimulation protects against pressure-induced damage through InsR-MAPK signaling in human trabecular meshwork cells. MOL MED REP 2017, 16(1):617-624.

37. Ahadome SD, Zhang C, Tannous E, Shen J, Zheng JJ: Small-molecule inhibition of Wnt signaling abrogates dexamethasone-induced phenotype of primary human trabecular meshwork cells. EXP CELL RES 2017, 357(1):116-123.

38. Webber HC, Bermudez JY, Millar JC, Mao W, Clark AF: The Role of Wnt/beta-Catenin Signaling and KCadherin in the Regulation of Intraocular Pressure. Invest Ophthalmol Vis Sci 2018, 59(3):1454-1466.

39. Zhou M, Diao Z, Yue X, Chen Y, Zhao H, Cheng L, Sun J: Construction and analysis of dysregulated IncRNA-associated ceRNA network identified novel IncRNA biomarkers for early diagnosis of human pancreatic cancer. Oncotarget 2016, 7(35):56383-56394.

40. Zhang Y, Xu Y, Feng L, Li F, Sun Z, Wu T, Shi X, Li J, Li X: Comprehensive characterization of IncRNAmRNA related ceRNA network across 12 major cancers. Oncotarget 2016, 7(39):64148-64167.

41. Li M, Ning J, Li Z, Fei Q, Zhao C, Ge Y, Wang L: Long noncoding RNA OIP5-AS1 promotes the progression of oral squamous cell carcinoma via regulating miR-338-3p/NRP1 axis. BIOMED PHARMACOTHER 2019, 118:109259.

42. Bai Y, Li S: Long noncoding RNA OIP5-AS1 aggravates cell proliferation, migration in gastric cancer by epigenetically silencing NLRP6 expression via binding EZH2. J CELL BIOCHEM 2019.

43. Wang M, Liu Y, Li C, Zhang Y, Zhou X, Lu C: Long noncoding RNA OIP5-AS1 accelerates the ox-LDL mediated vascular endothelial cells apoptosis through targeting GSK-3beta via recruiting EZH2. AM J TRANSL RES 2019, 11(3):1827-1834.

44. Gharesouran J, Taheri M, Sayad A, Mazdeh M, Omrani MD: Integrative analysis of OIP5-AS1/HUR1 to discover new potential biomarkers and therapeutic targets in multiple sclerosis. J CELL PHYSIOL 2019, 234(10):17351-17360.

45. Zhang J, Zhao T, Tian L, Li Y: LncRNA OIP5-AS1 Promotes the Proliferation of Hemangioma Vascular Endothelial Cells via Regulating miR-195-5p/NOB1 Axis. FRONT PHARMACOL 2019, 10:449.

46. Zhao J, Du X, Wang M, Yang P, Zhang J: Salidroside mitigates hydrogen peroxide-induced injury by enhancement of microRNA-27a in human trabecular meshwork cells. Artif Cells Nanomed Biotechnol 2019, 47(1):1758-1765. 
47. Wang X, Li Z, Bai J, Song W, Zhang F: miR175p regulates the proliferation and apoptosis of human trabecular meshwork cells by targeting phosphatase and tensin homolog. MOL MED REP 2019, 19(4):3132-3138.

\section{Figures}

A

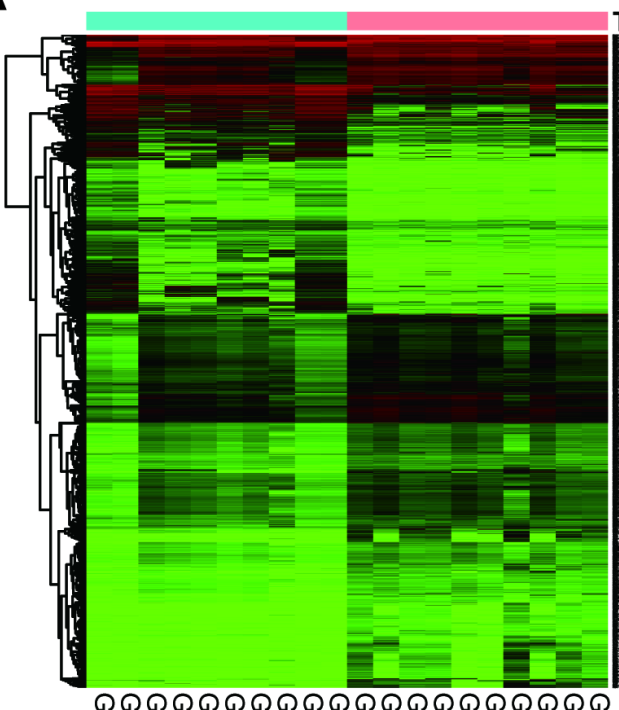

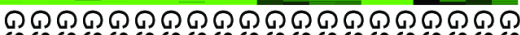
SW NonNnNenc

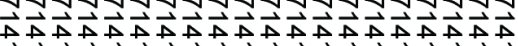

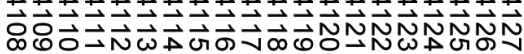

C

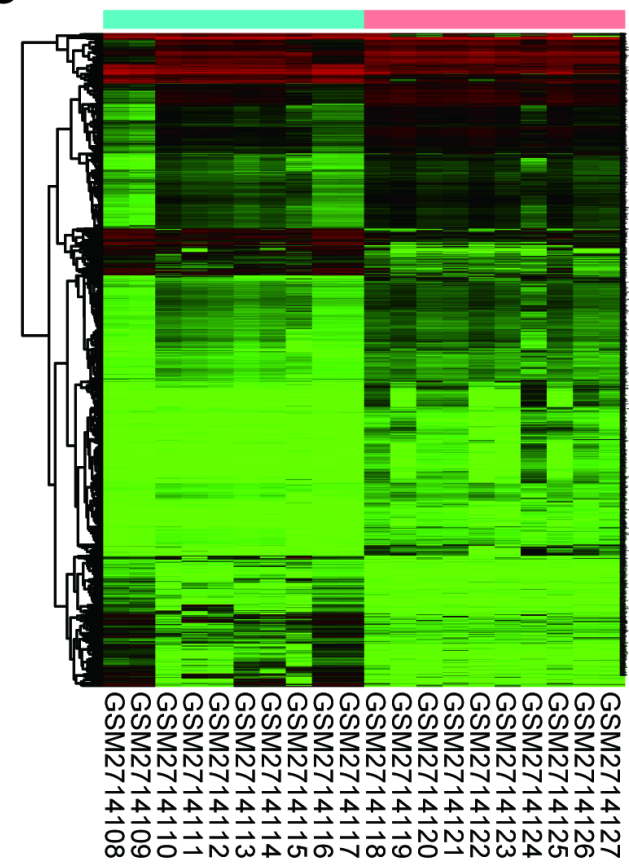

B

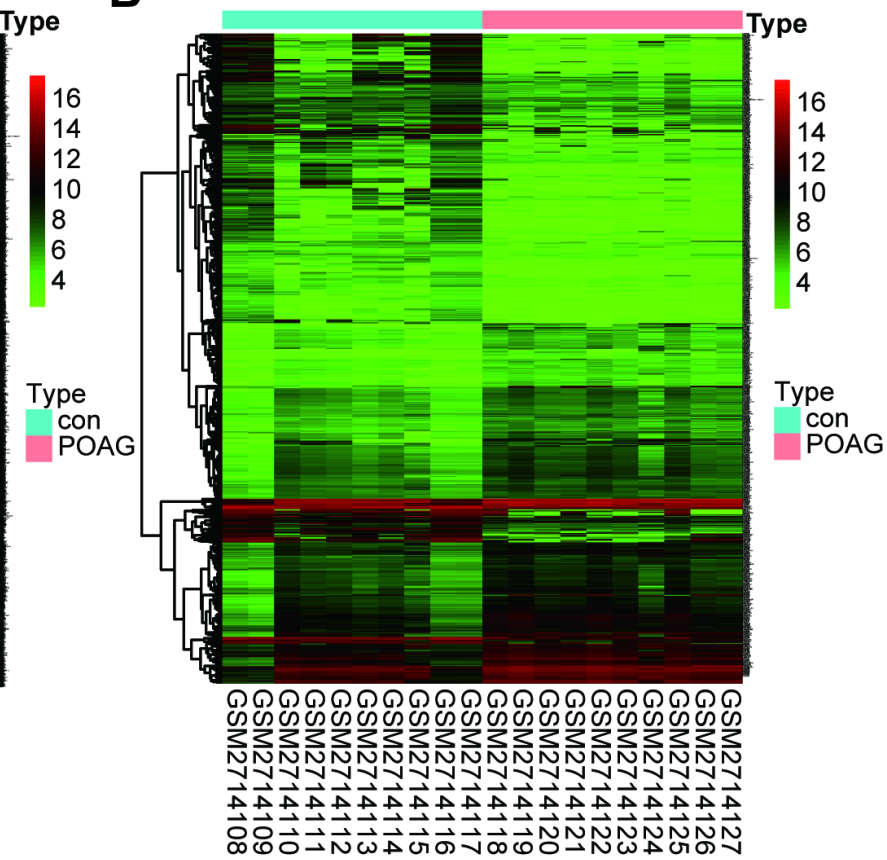

D

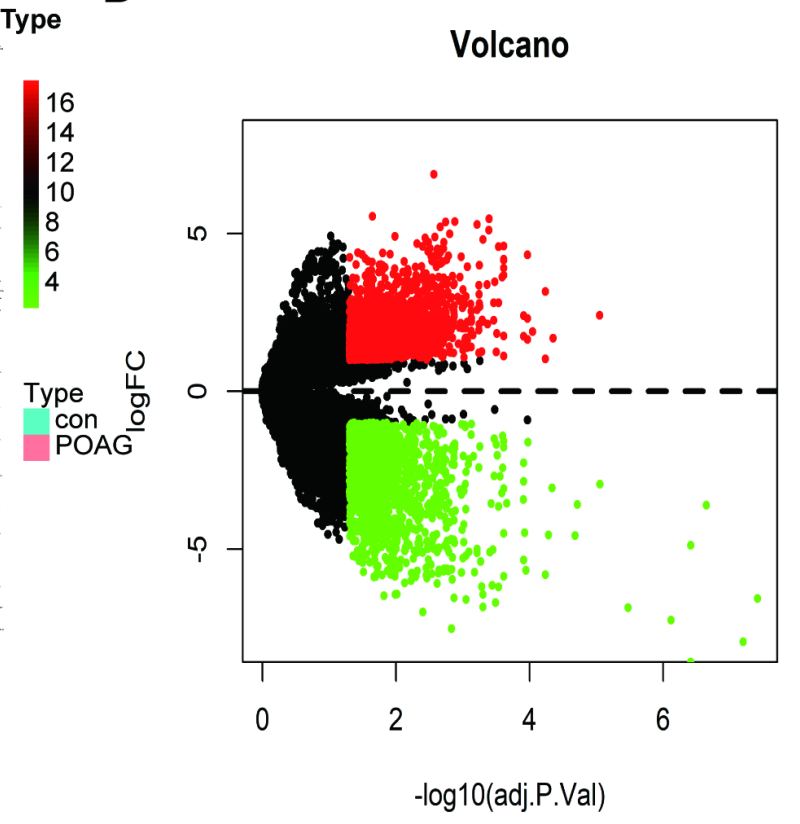


The differentially expressed RNAs in POAG. $\log 2 \mathrm{FC}>1$, false discovery rate $(F D R)<0.05$. A. Heatmap plots of differentially expressed RNAs. B. Heatmap plots of differentially expressed IncRNAs. C. Heatmap plots of differentially expressed mRNAs. The horizontal axis represents samples. The vertical axis represents RNAs. D. Volcano plot of differentially expressed RNAs in POAG.

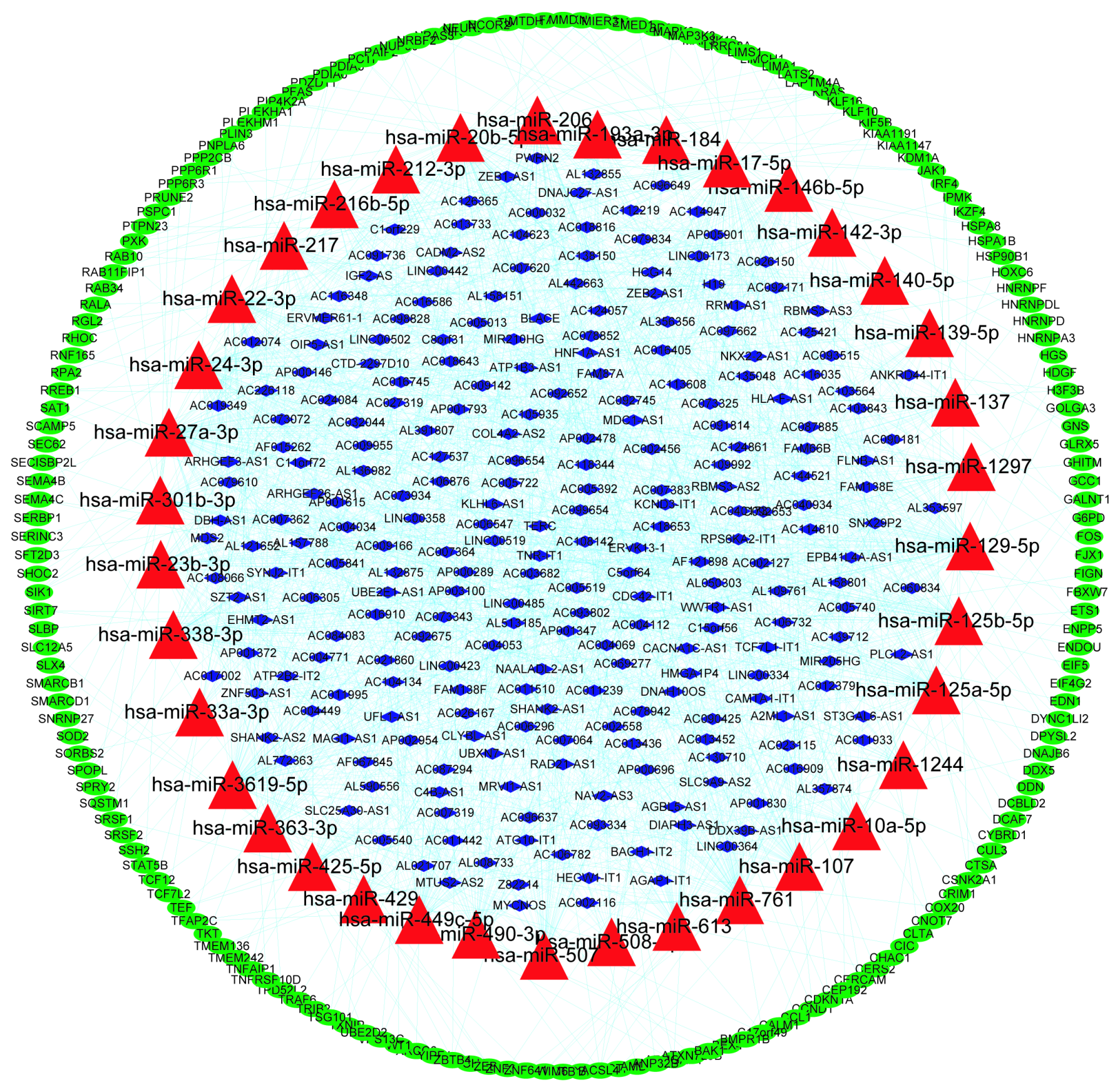

Figure 2

Overview of the IncRNA-miRNA-mRNA ceRNA network related with POAG. The blue diamonds, red triangles, and green ellipses nodes represented IncRNAs, miRNAs and mRNAs respectively. Blue lines 

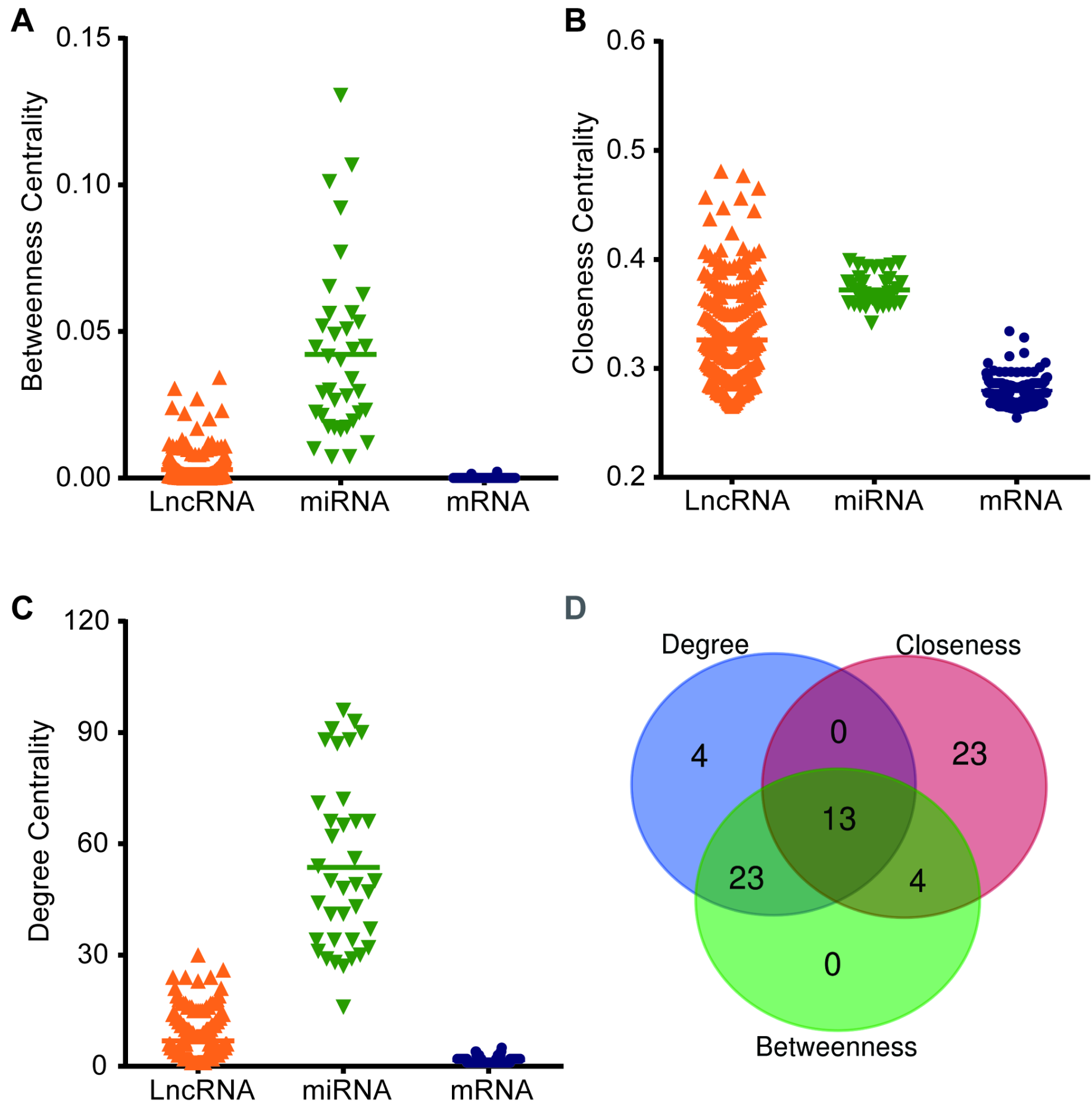

D

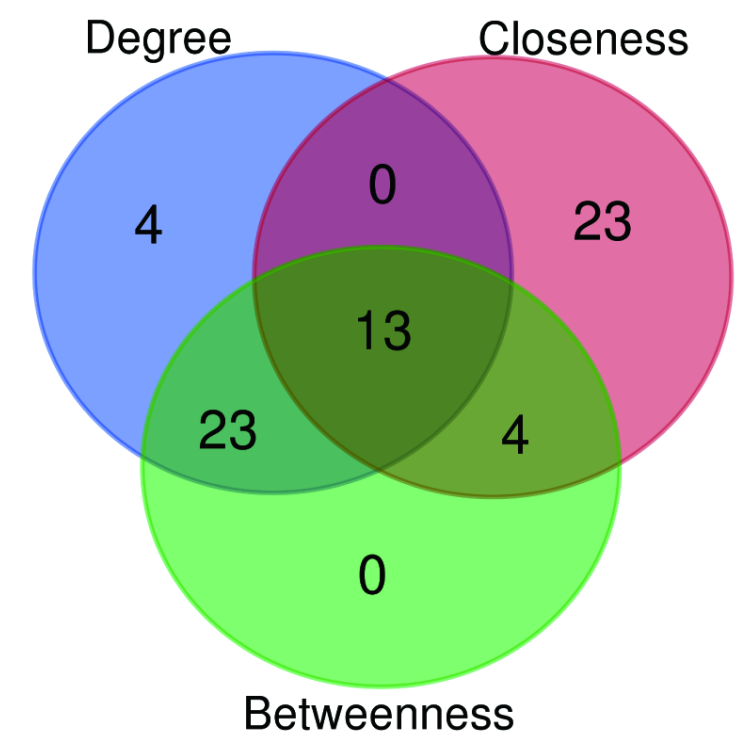

Figure 3

The difference in the betweenness, closeness, and degree centrality among IncRNAs, miRNAs, and mRNAs. A. The IncRNA nodes had a significantly higher betweenness centrality than mRNA nodes in the network. B. The IncRNA nodes had a higher closeness centrality than mRNA nodes in the network. C. The 
IncRNA nodes had a higher degree centrality than mRNA nodes in the network. D. The Venn diagram showed the overlap of top 40 genes with topological features in each dimension.
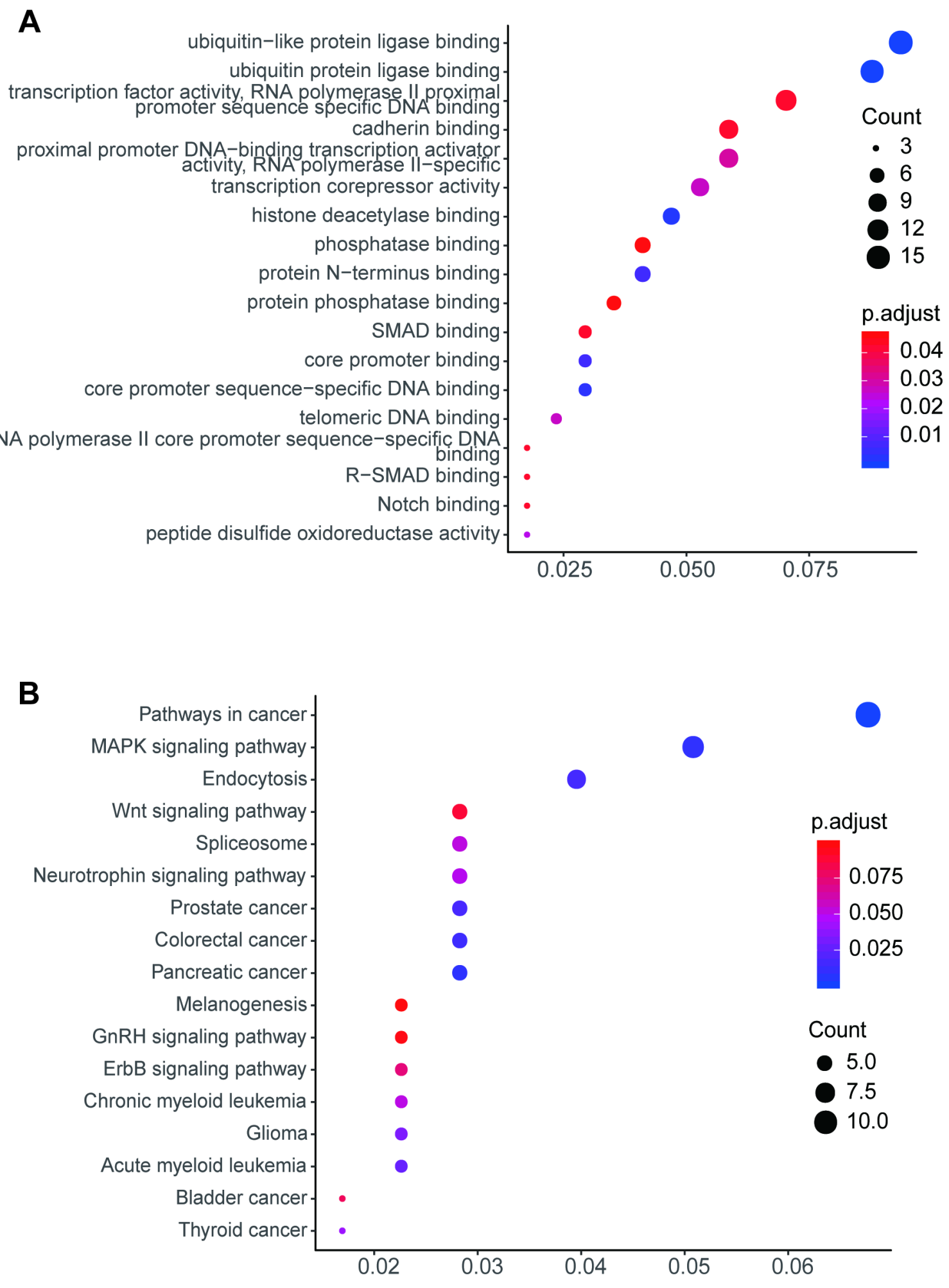

Figure 4

Gene ontology (A) and KEGG pathway (B) enriched analysis of the mRNAs in the ceRNA network. Y-axis label represents terms name, and $\mathrm{X}$-axis label represents gene ratio which is defined as the percentage of target genes per term. 